Research article

\title{
Title: Testosterone increases risk-taking for status but not for money
}

Losecaat Vermeer, A.B. ${ }^{1 *}$, Boksem, M.A.S. ${ }^{2}$, Gausterer, C. ${ }^{3}$, Eisenegger, C. $^{{ }^{+}}{ }^{\dagger}$, \& Lamm, $^{1}{ }^{1,4}$

${ }^{1}$ Neuropsychopharmacology and Biopsychology Unit, Department of Cognition, Emotion, and Methods in Psychology, Faculty of Psychology, University of Vienna, Vienna, Austria

${ }^{2}$ Rotterdam School of Management, Erasmus University, PO Box 1738, 3000 DR, Rotterdam, The Netherlands

${ }^{3}$ FDZ-Forensisches DNA Zentrallabor GmbH, Medical University of Vienna, Austria

${ }^{4}$ Vienna Cognitive Science Hub, University of Vienna, Vienna, Austria

$\left(^{*}\right)$ corresponding author: annabel.losecaat.vermeer@univie.ac.at

$(+)$ deceased

\section{Address:}

Neuropsychopharmacology and Biopsychology Unit, Department of Cognition, Emotion, and Methods in Psychology, Faculty of Psychology, University of Vienna, Liebiggasse 5, 1010 Vienna, Austria

Conflict of Interest: The authors declare no competing financial interests. 


\section{Abstract}

2 Testosterone has long been thought to increase risk-taking, but evidence supporting this association

3 is mixed. Instead, testosterone's key role may be to promote status-seeking behaviors. Here, we 4 examined to what extent testosterone administration affects risk preferences for both monetary and

5 social status outcomes, and whether this relationship is moderated by an individuals' social status.

6 Male participants $(N=166)$ experienced high or low status in a competition task and then played two

7 risk tasks; one involving gambles with only monetary outcomes, and another one involving gambles

8 with non-monetary outcomes that influenced their social rank. We found that testosterone (vs.

9 placebo) altered risk preferences for gains and losses in social rank, but not for monetary gains and

10 losses. Specifically, testosterone increased risk-taking to increase social rank in individuals with high,

11 but not low social status. These results demonstrate a context-dependent role of testosterone in 12 regulating risk-taking for social status.

\section{Keywords}

14 testosterone; social status; risk; decision making; dopamine; genetic polymorphism 


\section{Introduction}

Testosterone is a steroid hormone primarily associated with status-seeking behaviors such as aggression and competition (Eisenegger, Haushofer, \& Fehr, 2011). In parallel, there is growing interest for its proposed role in non-social behaviors such as risk-taking. A popular lay belief is that testosterone causes excessive risk-taking (e.g. reckless driving, and competition more generally), with news outlets even blaming the hormone for the financial crisis in 2008 (e.g. Scientific American, 2009). To date, the scientific literature has been relatively mixed about whether testosterone actually increases risktaking. A major remaining question is, under which circumstances testosterone causally affects risktaking, and if this depends on whether risk-taking can be a means to achieve higher social status or not.

Both in the field and laboratory experimental settings (reviewed in Apicella, Carré, \& Dreber, 2015; Stanton, 2017), testosterone levels correlated with changes in economic decision-making. For example, changes in testosterone predicted increased preference for risky choices involving monetary gains and losses (Apicella, Dreber, \& Mollerstrom, 2014). Other work found enhanced risk-taking, but only among individuals with high and low baseline testosterone levels (Stanton et al., 2011), and testosterone to influence risk-taking only in individuals with low baseline cortisol levels (Mehta, Welker, Zilioli, \& Carré, 2015). Nonetheless, there have also been reports of null results (e.g., Derntl, Pintzinger, Kryspin-Exner, \& Schöpf, 2014).

Of the several investigations on the causal influence of exogenous testosterone on risk-taking, only half reported positive or mixed evidence (Cueva et al., 2015; Goudriaan et al., 2010; Nadler, Jiao, Johnson, Alexander, \& Zak, 2017; van Honk et al., 2004; Wu et al., 2016), whereas others found no statistically significant effect (Boksem et al., 2013; Zethraeus et al., 2009). In sum, both correlational and testosterone administration studies demonstrate that testosterone's influence on risk-taking remains debated.

Risk-taking may function as a means for attaining higher social status (Mehta, Welker, et al., 2015). For instance, individuals' relative status has been shown to influence risk-taking in a competition to 
gain a higher status (Ermer, Cosmides, \& Tooby, 2008). One proposal is that testosterone may function to enhance risk-taking to attain higher social status, but not or to a lesser degree when risk-taking does not directly serve one's social status.

Theoretical models of testosterone (Archer, 2006; Mazur \& Booth, 1998) postulate that testosterone fluctuates with social challenges and predicts status-relevant risky behaviors, such as aggression (reviewed in Geniole \& Carré, 2018), competition (Casto, Edwards, Akinola, Davis, \& Mehta, 2020; Losecaat Vermeer et al., 2020; Mehta \& Josephs, 2006; Mehta, Son, et al., 2015), and social trust (Boksem et al., 2013). While some work controlled for individual risk-taking by using a separate nonsocial task (Boksem et al., 2013; Eisenegger, Kumsta, Naef, Gromoll, \& Heinrichs, 2017), no studies to date tested whether testosterone alters risk-taking with direct status implications, and whether this depends on individuals' social status levels (see Fisk, Miller, \& Overton, 2017).

According to the Biosocial Model of Status (Mazur \& Booth, 1998), changes in testosterone following a win (i.e. equivalent to high status) may reinforce status-enhancing risky behaviors, whereas changes following a loss (i.e. equivalent to low status) may inhibit status-enhancing behaviors. This directly motivated the risk-for-status hypothesis in the present investigation. Extending on previous theories of the effects of testosterone, we predicted that high testosterone in individuals with high social status increases risk-taking when this could serve attaining higher status, whereas high testosterone in individuals with low social status should not, or even reduce risk-taking that can impact their social status. Importantly, these considerations would also predict that testosterone only influences risk-taking when it has direct implications for social status, and not when risk-taking has no implications to status.

To test this, we performed a double-blind placebo-controlled administration study in which men received either testosterone or placebo, and played a competition task where their social status was manipulated to be either high or low, indicated via a ranking. Following the competition, participants played two gambling tasks. The first task only contained monetary gain-loss gambles with no impact to their social rank. The second task contained gambles with gain-loss outcomes in social rank without 
monetary incentives, to assess risk-taking for social status independent of monetary reward. The present study is thus the first to simultaneously manipulate testosterone and status to test their effects on risk-taking, while additionally dissociating risk preferences for money and for status.

Testosterone effects on status-seeking behaviors may depend on the functioning of the testosterone-binding androgen receptor (Eisenegger et al., 2017; Geniole et al., 2019; Losecaat Vermeer et al., 2020), and testosterone's actions on the striatal dopaminergic reward system (Losecaat Vermeer, Riečanský, \& Eisenegger, 2016; Mehta, Son, et al., 2015). We thus explored whether effects of testosterone on risk-taking are also moderated by individual genetic differences, focusing on polymorphisms related to the androgen receptor, and striatal dopamine. The efficiency of the androgen receptor is negatively correlated with the cytosine-adenine-guanine (CAG)-repeat length in exon 1 of the androgen receptor gene (Zitzmann \& Nieschlag, 2003), therefore we determined CAGrepeat length for each participant. Moreover, testosterone increases dopamine and activity in the ventral striatum, in animals (de Souza Silva, Mattern, Topic, Buddenberg, \& Huston, 2009) and humans (Hermans et al., 2010). We thus measured a polymorphism related to DAT1 (Heinz, 2000), the dopamine transporter, as a proxy for striatal dopamine.

\section{Method}

\section{Participants}

One hundred-seventy-three male volunteers participated in the study. This sample size was directly informed by previous high-profile single-dose testosterone administration studies on status-relevant behavior and risk-taking (e.g. Boksem et al., 2013; Carré et al., 2017; Eisenegger, Naef, Snozzi, Heinrichs, \& Fehr, 2010), and work on status-seeking behavior in relation to CAG-repeat polymorphism (Eisenegger et al., 2017). Participants were pre-screened and had no history of psychiatric, neurological, or endocrine disease, nor were currently using or had been regular users of marijuana or any psychotropic drugs and corticosteroids, and had not taken part in a medical study in the four weeks preceding the study. The pharmacokinetics of testosterone gel administration has been established in 
men (Eisenegger, von Eckardstein, Fehr, \& von Eckardstein, 2013), therefore our sample only included men. Seven participants were excluded; six participants due to a technical error, which resulted in either a partial or complete loss of their data $(n=2)$, or in incorrect rank presentation $(n=4)$, and one participant who was unintentionally included twice in the study. The final sample consisted of 166 men (Placebo $(N=84): M=24.55$ years, $S D=3.64$; Testosterone $(N=82): M=24.38$ years, $S D=3.33)$. All participants provided consent and were financially compensated for completing the study. The study procedure was performed in accordance with the Declaration of Helsinki and approved by the Medical Ethics Committee of the University of Vienna (1918/2015).

\section{General procedure}

Participants arrived to the lab on two separate days. On the first day, participants filled out a series of personality questionnaires including demographics, provided DNA for genotyping, and were measured for their Body Mass Index (BMI) and body fat using a body composition monitor (Omron BF51). At the end of the day, participants received 10 Euro endowment in advance to enhance the feeling of ownership of this money which they could later lose in the subsequent experiment. They were instructed that this endowment was theirs to keep and to pay for any losses during the risk task on the second test day.

The second day, at 10:00 a.m. participants arrived to the lab in groups of nine. After participants provided consent and received instructions of the procedure, they completed additional questionnaires assessing self-reported mood and personality traits such as trait dominance (16-PF-R, Schneewind \& Graf, 1998), which has previously shown to moderate effects of testosterone on statusseeking, mainly in individuals with high trait dominance (Mehta, Son, et al., 2015). Twenty minutes after arrival, participants provided the first saliva sample $\left(t_{0}\right)$, to assess baseline hormone concentrations. Next, participants were administered with testosterone or placebo by random assignment (section Drug administration). Participants waited in the lab for two hours to allow the drug to reach peak level, but did not interact with others. Two-hours post-administration, participants 
completed a second mood questionnaire to control for a potential confound of treatment, see Supplementary Online Material (SOM), and provided their second saliva sample $\left(\mathrm{t}_{120}\right)$. To minimize diurnal hormone variability, the behavioral testing occurred from 1:00-3:00 p.m. Testing included a competition paradigm with a brief post-task questionnaire (Losecaat Vermeer et al., 2020), followed by the risk paradigm and then one other task which is unrelated to the question reported here. At the end, we assessed participants' beliefs about whether they believed they had received testosterone or placebo via a binary choice, and their confidence about their answer on a scale from 0-100 (Table 1). After that we paid them for participation and paid out any gains from the risk task.

Table 1. Descriptives by treatment (Placebo, Testosterone)

\begin{tabular}{lcccccccc}
\hline & \multicolumn{3}{c}{ Placebo $(\boldsymbol{N}=\mathbf{8 4})$} & \multicolumn{3}{c}{ Testosterone $(\boldsymbol{N}=\mathbf{8 2})$} & \multicolumn{2}{c}{ Statistics } \\
\cline { 2 - 10 } Age (years) & $n$ & $M$ & $S D$ & $n$ & $M$ & $S D$ & $t$-value & $p$-value \\
\cline { 2 - 10 } BMI & 84 & 24.55 & 3.64 & 82 & 24.38 & 3.33 & 0.314 & .754 \\
Trait Dominance & 80 & 24.27 & 3.35 & 82 & 24.12 & 2.69 & 0.331 & .741 \\
Testosterone $\log (\mathrm{ng} / \mathrm{mL})-\mathrm{t}_{0}$ & 84 & 42.52 & 24.17 & 82 & 45.82 & 25.78 & -0.849 & .397 \\
Testosterone $\log (\mathrm{ng} / \mathrm{mL})-\mathrm{t}_{120}$ & 84 & 0.08 & 0.36 & 77 & 0.05 & 0.43 & 0.447 & .656 \\
Cortisol $\log (\mathrm{ng} / \mathrm{mL})-\mathrm{t}_{0}$ & 82 & 0.33 & 0.49 & 80 & 3.63 & 1.53 & -18.48 & $<.001$ \\
Cortisol $\log (\mathrm{ng} / \mathrm{mL})-\mathrm{t}_{120}$ & 84 & -0.18 & 0.69 & 76 & 0.58 & 0.70 & 0.269 & .789 \\
Drug belief $(\%)$ & 84 & 0.24 & 0.43 & 82 & 0.23 & 0.42 & 0.096 & .923 \\
Drug belief confidence $(0-100 \%)$ & 84 & 56.40 & 25.24 & 82 & 55.39 & 26.39 & 0.253 & .801
\end{tabular}

Note: for some comparisons $n$ slightly differs from the total $N$ of each group, i.e. some saliva samples were unanalyzable, and four BMI scores of the placebo group were not recorded. Drug belief $(\%)=$ percentage of participants who believed they had received testosterone, $t_{0}=$ baseline, $t_{120}=$ post-administration .

\section{Task procedure}

Prior to the risk task, we experimentally induced high or low social status in participants via a competition task (Losecaat Vermeer et al., 2020), which included nine players and lasted for approximately 60 minutes. In brief, the competition paradigm entailed a reaction time task, in which participants had to respond as fast as possible to a target on screen appearing at random intervals. The faster participants responded, the more points they could earn. Participants repeatedly played against each other and based on their score on each round they saw they obtained either a high (varying between 1-4) or low (varying between 6-9) position in a ranking list. Unbeknownst to 
participants, in reality their ranks were based on random assignment. Participants sat in the same room, in individual cubicles to make the competitive task more realistic.

Following the competition, participants performed the risk paradigm, consisting of two tasks. In the first task, participants performed a series of gambles involving monetary gains and losses. In the second task, participants performed a series of gambles involving social gains and losses (increases or decreases in the social ranking), without monetary outcomes. Participants were briefly reminded about their final position in the ranking resulting from the previously completed competition task, before receiving instructions and practice trials to perform the monetary risk task (Fig. 1a). Next, for the status risk task, we explicitly instructed participants that the outcome of the upcoming gambles would only affect their rank position by adding or deducting points from their score achieved in the competition, and could result in gain or loss in rank respectively. To compare high and low ranked participants, we presented participants with a new ranking consisting of players whom had previously participated in the experiment and had the same rank position. Specifically, both high and low-ranked participants were compared to eight participants with an identical rank position to themselves. In this new ranking, the name of each player and their score was shown, including the difference in points compared to the participant, who was placed in the middle (i.e. $5^{\text {th }}$ ). This was done to provide the opportunity to enhance and reduce further status by gaining or losing position in the ranking based on the outcome of these gambles. This new rank list was shown at all times at the top of the screen (Fig. 1b).

\section{Risk paradigm}

We developed a novel paradigm consisting of a monetary and status risk task in fixed order. For both, participants had to choose whether to accept or reject a gamble with an equal probability to win or lose. If participants decided to reject the gamble they would simply not gain or lose anything. No immediate feedback was given to avoid carry-over effects of the outcome to the next trial. At the end 
170

of the experiment, one gamble of each task was randomly chosen and played in case the participant had chosen to accept the gamble.

a

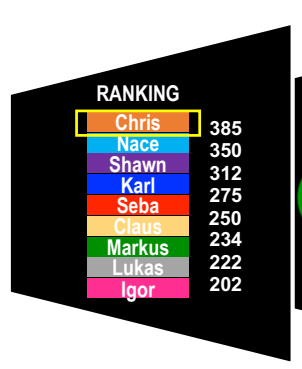

(5s)
Monetary gambles

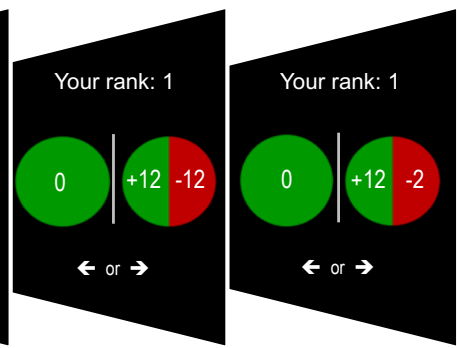

(self-paced) b

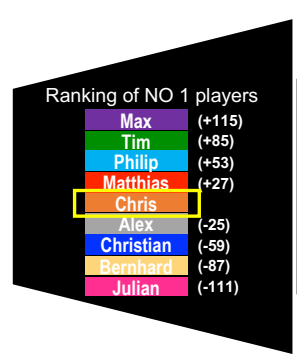

(5s)
Status gambles

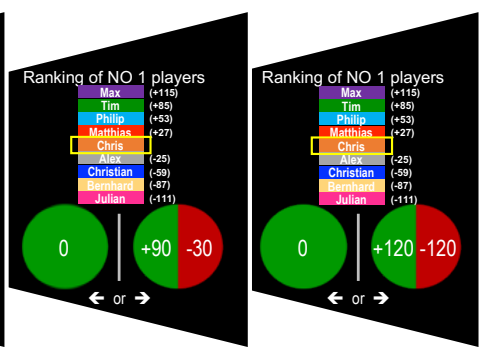

(self-paced)
171

172

173

174

175

176

177

178

179

180

181

182

183

184

185

186

187

188

189

190

191

192

Figure 1. Risk paradigm. (a) Example of a monetary risk trial of a high ranked participant. After competition participants saw their name in the rank list. Followed by this rank list, participants could decide to accept the gamble with equal probability to win or lose or reject it (i.e. choose $€ 0$ ). (b) Example of a status risk trial of a high ranked participant. At the beginning, the participant saw a new ranking, consisting of players who had previously participated in the experiment and ended up in the participants' rank category (i.e. here high). While the participant's precise rank is number one, his performance is ranked $5^{\text {th }}$ among the other players of his rank category (i.e. whom also ended up as number one in their competition). Participants could decide to accept or reject gambles with equal probability to win or lose status points for rank position. No immediate feedback was provided in both tasks.

\section{Monetary risk task}

Sixteen trials, each containing a monetary gain/loss gamble were presented. The gambles had a fixed gain of $€ 12$ and a loss ranging from $€-1$ to $€-16$, with steps of $€ 1$ (Fig. 1a). On each trial, participant's rank was shown at the top of the screen. However, participants were instructed that the outcome of these gambles only had consequences for their monetary endowment of $€ 10$, but not for their status in the experiment.

\section{Status risk task}

Sixteen trials, each containing a gain/loss gamble in terms of points were presented. The gain and loss points ranged from 30 to 120 (in steps of 30 points) and had consequences for participants' position in the rank list. Points would be either added or subtracted from the participants' score depending on 
the gain or loss outcome and determined their final social status in the experiment. On each trial, the players' rank, including their score and difference in points to the participant's score was shown, for the participants to see the potential impact a specific gamble would have on their position on the ranking. The task also contained two gambles with an expected value of zero, but one with high and one with low variance (i.e. risk level). The high variance gamble provided a gain or loss of 120 points (i.e. gain/loss of 4 positions, resulting in first or last place), whereas the low variance gamble provided a gain or loss of 30 points (i.e. gain/loss of 1 position, resulting in $4^{\text {th }}$ or $6^{\text {th }}$ place respectively). There were also two catch trials to check if participants paid attention in which with an equal probability one could win 1 position or lose none, or lose 1 position or win none. Catch trials were excluded from analysis. The outcomes of the status gambles had no monetary consequences throughout, neither immediately nor later in the experiment.

\section{Drug administration}

We randomly assigned participants to receive a single dose of $150 \mathrm{mg}$ testosterone (Testoge $\mathrm{l}^{\circledR}, N=82$ ) or placebo gel $(N=84)$ in a double-blind procedure. The gel was topically applied to chest, shoulders and upper arms by a male research assistant. The pharmacokinetics of this dosage and administration technique have previously been established in men (Eisenegger et al., 2013). This showed testosterone levels to peak $3 \mathrm{~h}$ post-administration with a significant increase after $2 \mathrm{~h}$. Therefore, behavioral testing commenced $2 \mathrm{~h}$ post-administration, with the risk task 195 min post-administration. Recent work using larger samples and same dosage observed a significant increase in testosterone levels as fast as $1 \mathrm{~h}$ post-administration (see e.g. Carré et al., 2017). Notwithstanding, evidence of these studies when taken together suggest that testosterone levels were still sufficiently high in our testing window.

\section{Hormonal assays}

Hormone samples were collected via passive drool into a $2 \mathrm{~mL}$ polypropylene vial and stored at -30 degrees Celsius. Saliva samples were analyzed for testosterone and hydrocortisone with liquid 
chromatography tandem mass spectrometry (LCMS/MS) (see SOM for details). Saliva values were winsorized based on three median absolute deviations from the median and log-transformed. Table 1 shows baseline hormone concentrations $\left(t_{0}\right)$ did not differ between treatment groups, while testosterone concentration after administration $\left(t_{120}\right)$ was significantly increased in the testosterone vs. placebo group, confirming our manipulation was successful.

\section{Genotyping}

DNA was collected using sterile cotton buccal swabs (Sarstedt AG, Germany), and purified applying the QIAamp DNA Mini kit (Qiagen, Germany). CAG-repeat and DAT1 VNTR polymorphisms were investigated by multiplex PCR with fluorescent-dye-labeled primers and capillary electrophoresis (detailed in SOM). Treatment groups (Testosterone $M=22.11, S D=3.12$, Placebo $M=22.44, S D=2.80$ ) did not differ in mean CAG-repeat length $(\mathrm{t}(161.32)=0.72, p=.474)$. For the DAT1 VNTR polymorphisms, 9/10-repeat carriers (9/10R: 34\%; testosterone $n=29$ placebo $n=27$ ) and 10/10-repeat carriers (10/10R: $57 \%$; testosterone $n=48$, placebo $n=46$ ) occurred most in our sample (see also SOM). Therefore we used these two genotypes in the analyses, with 10/10-Repeat carriers assumed to have higher DAT, expression and hence lower striatal dopamine than 9/10-Repeat carriers (Heinz, 2000).

\section{Statistical analysis}

To assess whether testosterone administration alters risk-taking, we ran a generalized mixed effects model on the decision to accept or reject the gamble, using Ime4 (Bates, Mächler, Bolker, \& Walker, 2015) in the R statistical software package (version 3.4.3, R Development Core Team, 2017). The model included between-subject predictors (at level two) for Drug (testosterone, placebo) and Rank (high, low), and a within-subject predictor (at level one) for Expected Value of the gamble, including their interactions. The model also included a random intercept for participant including by-participant random slopes for expected value of the gambles to account for repeated observations and variability among participants. We ran this model separately on the behavior for the monetary gamble task and 
for the status gamble task, because these were played independently in a fixed order and had different magnitudes and expected values, and thus were not entirely comparable. $\mathrm{P}$ values for regression coefficients were determined with Likelihood Ratio Tests. Reported estimates are odds ratios (OR) and Wald 95\% confidence intervals (Cls), and means are estimated marginal means unless otherwise stated. Planned and post-hoc comparisons were obtained using emmeans, based on two-tailed tests where no other statistical corrections (e.g., adjustments for multiple comparisons) were made. The total sample broken down for each group: Testosterone $(n=82)$ including high rank $n=45$ and low rank $n=37$; Placebo ( $n=84)$ including high rank $n=40$, low rank $n=44$.

\section{Moderators}

Baseline cortisol, trait dominance, CAG-repeat and DAT1 polymorphisms were separately included in the model as described above, to explore whether they influenced the effect of testosterone on risktaking depending on social status. Expected value of the gambles was included as predictor and random effect only, without interactions, to reduce model complexity, which was supported by model comparison (full model incl. interactions, $\chi^{2}(3)=4.76, p=.199$ ).

\section{Results}

\section{Social rank influences monetary risk-taking, independent of testosterone}

Participants with a low rank position showed increased risk-taking for monetary gambles as compared to those with high $\operatorname{rank}$ (low $\operatorname{rank} M=35.7 \%, 95 \% \mathrm{Cl}=[21.9 ; 52.4]$, high $\operatorname{rank} M=13.8 \%, 95 \% \mathrm{Cl}=[6.9$; 23.5], $p=.007$, Table 2, Fig. 2a). As shown in Table 2, testosterone (vs. placebo) did not differentially influence risk preferences for monetary gambles, nor was this influenced by whether participants had a high and low rank (drug $x$ rank, $p>.05$, Fig $2 b$ ). Participants accepted more gambles with increasing expected value (Fig. 2c), but this was not significantly moderated by testosterone or the participant's rank (Table 2). 
273

a

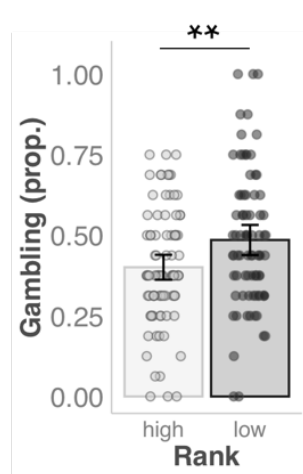

b

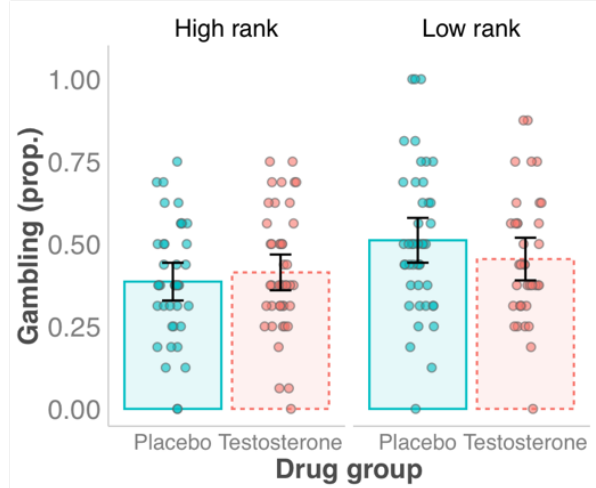

C

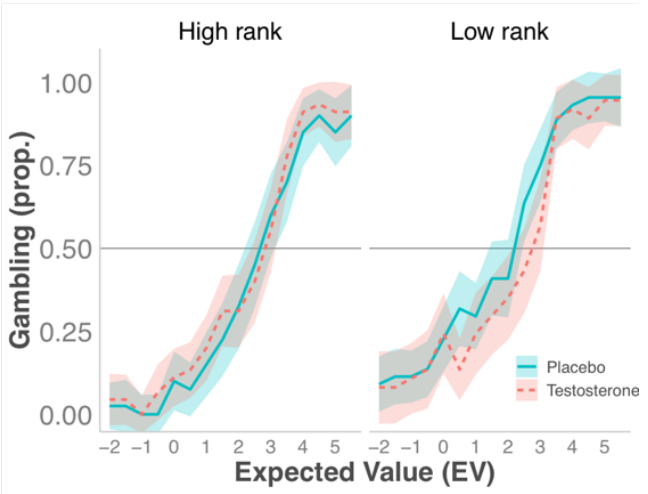

274

275

276

277

278

279

280

281

282

283

284

285

286

Figure 2. Mean (raw) proportion risk-taking for monetary outcomes. (a) Risk-taking for low compared to high rank category. Every dot represents participants' mean. (b) Risk-taking by drug group (testosterone or placebo) of high and low rank showed no significant interaction. (c) Risk-taking by expected value of the gamble (x-axis, range -2 to 5.5), for drug and rank. The horizontal line represents the point of indifference (i.e. the expected value where proportion of gambling is 0.50$)$. Testosterone had no statistical significant effect on risk-taking by rank and expected value. Error bars and shaded bands represent $95 \%$ confidence intervals. Asterisks indicate a significant difference between rank $(* * p<.01)$.

Table 2. Results of generalized mixed effects models for Money and for Status, involving Drug (testosterone, placebo), Rank (high, low) and Expected Value (EV)

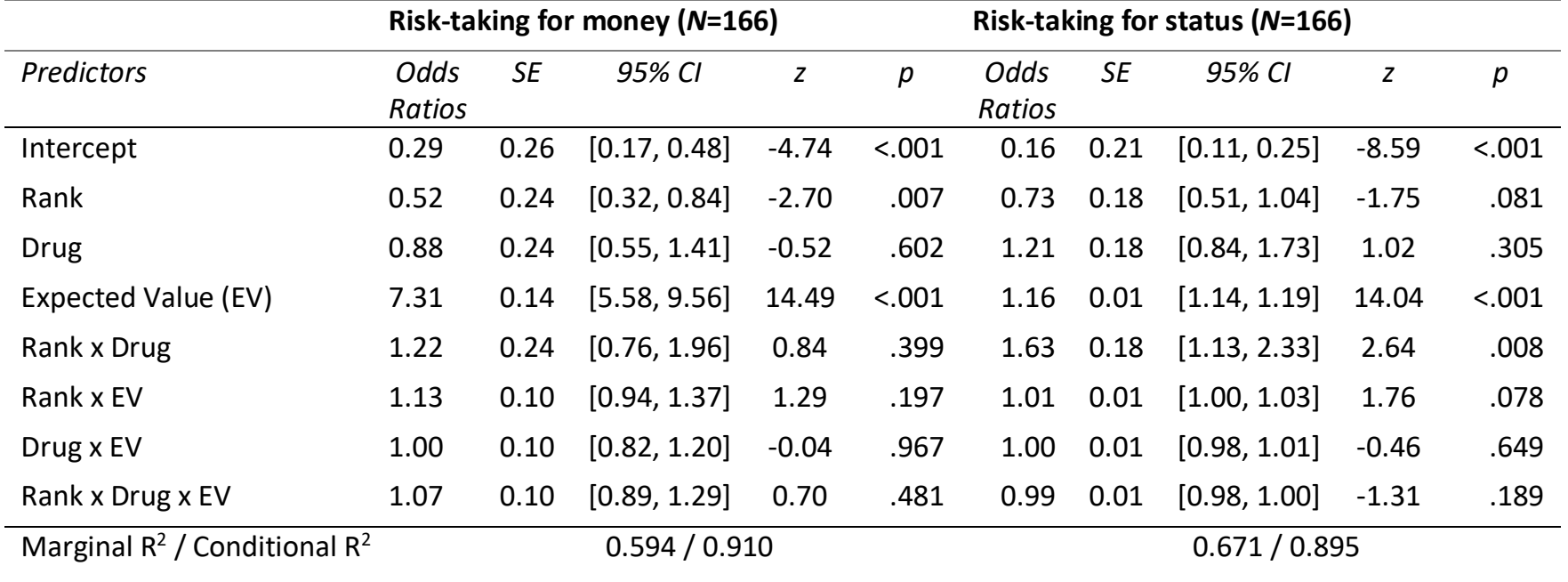

287 Note: sum-to-zero contrasts were applied (i.e. effect coding). Testosterone and High rank were coded as 1.

288 Expected Value was centered at 0.

\footnotetext{
${ }^{1}$ Including baseline cortisol or DAT1 resulted in a marginal effect of rank ( $p=.081, p=.0506$, respectively).
} 


\section{Testosterone increases risk-taking for status outcomes in individuals with high social status}

Testosterone influenced risk-taking for status depending on participants' rank (Table 2), such that testosterone (vs. placebo) increased risk-taking in participants with a high rank $(\mathrm{OR}=3.85,95 \% \mathrm{Cl}=$ $[1.36,10.92], z=2.10, p=.036)$, but not in participants with a low rank $(\mathrm{OR}=0.55,95 \% \mathrm{Cl}=[0.20$, 1.49], $z=-1.07, p=.285$, Fig. 3a). Rank had a similar effect on risk-taking for status as on risk for money, but this just failed to reach significance $(p=.081)$. Participants accepted more gambles with increasing expected value, though no interaction with drug or rank was observed (Table 2, Fig. 3b).

a

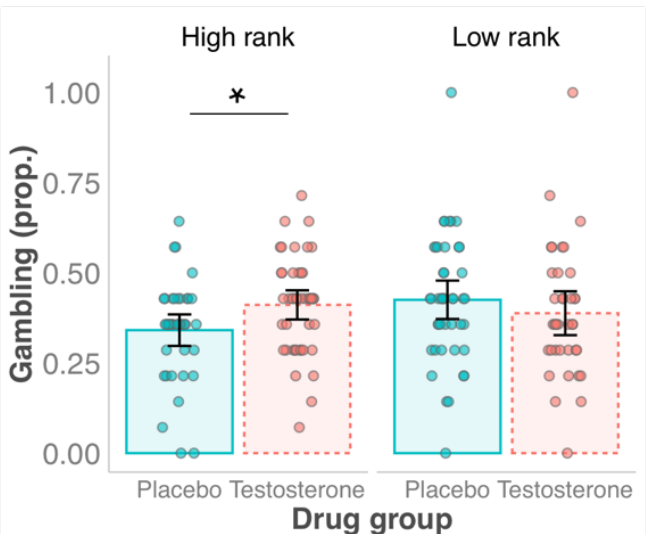

b

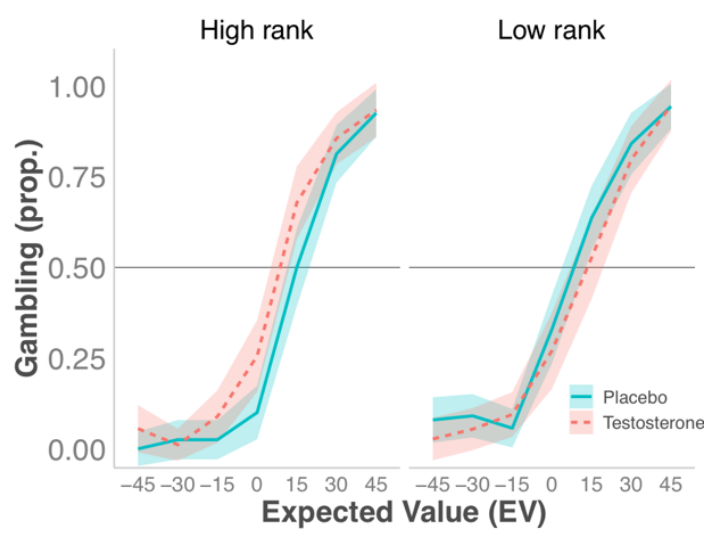

Figure 3. Mean (raw) proportion risk-taking for status. (a) Risk-taking for each drug group (testosterone or placebo) of high or low rank. Testosterone (vs. placebo) increased risk-taking in participants of high rank, but not low rank. Every dot represents participants' mean. (b) Risk-taking by expected value of the gamble ( $x$-axis), for drug and rank. Expected value influenced risk-taking, but not differentially for drug or rank. The horizontal line represents the point of indifference (i.e. the expected value where proportion of gambling is 0.50 ). Error bars and shaded bands represent $95 \%$ confidence intervals. Asterisks indicate a significant difference between drug $(* p<.05)$.

Baseline cortisol, trait dominance, CAG-repeat and DAT1 polymorphisms did not interact with drug and rank to predict risk-taking ${ }^{2}$. Despite a nonsignificant interaction, when observing the data of high and low cortisol groups (based on median split), the conditional effect of testosterone seemed more pronounced in individuals with low baseline cortisol (for details see SOM). However, testosterone influenced risk-taking depending on DAT1 genotype $(95 \% \mathrm{Cl}=[0.48,0.97], p=.035)$, with

\footnotetext{
2 The main drug $x$ rank interaction changed to marginal significance when including trait dominance $(p=.056)$ or DAT1 $(p=.081)$, but drug $\times$ rank interaction was significant in all other models. Importantly, including all four as covariates in one model demonstrated that the drug $\mathrm{x}$ rank interaction effect was robust $(p=.024$, see SOM $)$.
} 
testosterone (vs. placebo) appearing to increase risk-taking in 10/10-carriers, and reduce risk-taking in 9/10-carriers.

\section{Discussion}

In the current study we investigated whether exogenous testosterone influenced risk-taking for choices that could alter the individual's social status level, and for choices with pure monetary outcomes, and whether this was dependent on the individual's current social status (i.e. high or low rank). This was mainly motivated by previous mixed results regarding the influence of testosterone on risk-taking, which had not accounted for the potentially important moderating role of social status. Based on prevailing models and research on testosterone function and status-seeking (Archer, 2006; Mazur \& Booth, 1998), we formulated a risk-for-status hypothesis that predicted testosterone to influence risk-taking when this could serve to attain or maintain high social status. Conversely, this hypothesis predicted that testosterone would not (or less so) influence risk-taking that does not provide an opportunity to improve social status (e.g. gambling to only gain money).

Results confirmed our risk-for-status hypothesis. Exogenous testosterone (vs. placebo) enhanced risk-taking for status in individuals with high social rank, but not in individuals with low social rank. Importantly, monetary risk-taking was unaffected by testosterone administration. Our findings crucially add to previous theories and studies on testosterone and status-seeking (Eisenegger et al., 2011), by showing that testosterone influenced risk preferences for options with a possible impact to social status in men with a high social rank, but not for options with monetary outcomes without direct status consequences.

Our findings are of relevance for previous research reporting an overall positive but weak association of testosterone and monetary risk-taking (Cueva et al., 2015; Goudriaan et al., 2010; Mehta, Welker, et al., 2015; Nadler et al., 2017; van Honk et al., 2004; Welker, Roy, Geniole, Kitayama, \& Carré, 2019). Besides differences in methodologies and sex/gender of the samples (previous work mainly used female participants), most of the studies have not manipulated the relative social status 
or accounted for the implications for one's social status as a contextual factor in their design and analysis. Therefore, one possible reason for the previously reported inconsistencies in the literature for testosterone and risk-taking is whether individuals could influence their social status through risktaking. In the current study, we explicitly told participants that the monetary gambles would not influence their status level, thereby excluding any perceived challenge or opportunity to improve status. By combining a pharmacological intervention with a risk task containing gambles with status outcomes, we showed that the social implications of choices are an important moderator of testosterone function in risk-taking. We therefore recommend future studies to consider social status in their design and analysis, to further elucidate testosterone's role in risk-taking.

Low social status has in previous studies been associated with increased monetary risk-taking (Welker, Goetz, \& Carré, 2015). Likewise, we found increased risk-taking for monetary gambles in individuals with an experimentally manipulated low compared to high social status. Importantly, their status level was irrelevant to the choices at hand. Previous research for monetary choices has shown that people typically avoid risk following a gain, but take risk following a loss, even when these prior gain and loss outcomes were unrelated to the current choice (Losecaat Vermeer, Boksem, \& Sanfey, 2014; Tversky \& Kahneman, 1981). Relatedly, the anticipation of a social loss increased risk-taking (i.e. overbidding) in an experimental auction (Delgado, Schotter, Ozbay, \& Phelps, 2008). Our results add to these findings, demonstrating that a high or low social status influenced risk preferences in a similar direction as previously experienced monetary losses. Importantly, this was the case even though status information had no relevance to the decision.

Previously identified moderators of testosterone (i.e. trait dominance, baseline cortisol, CAGrepeat) did not influence effects of testosterone and rank on risk-taking in the present experiment. Although we found no statistical interaction with basal cortisol, applying a median split, the conditional effect of testosterone on risk-raking for status tends to be more pronounced in individuals with low cortisol, consistent with predictions of the dual-hormone hypothesis (Mehta, Welker, et al., 2015). In contrast to previous work on aggression and competition (Geniole et al., 2019; Losecaat Vermeer et 
al., 2020), we found no association between high androgen receptor sensitivity to testosterone (CAGrepeat) and status-driven risk-taking. DAT1-genotype (hypothesized to affect striatal dopamine availability) influenced risk-taking for status depending on drug administration. While risk-taking was significantly different between DAT1-genotype individuals under placebo, testosterone administration appeared to reverse this effect. Testosterone increased risk-taking in 10/10R-carriers (i.e. lower endogenous dopamine), and to a smaller degree reduced risk-taking in 9/10R-carriers (i.e. higher endogenous dopamine), although this was nonsignificant at post-hoc level. Previous research demonstrated increased striatal activity during reward anticipation following testosterone administration, specifically in low intrinsically motivated women (Hermans et al., 2010). Consistent with this, our findings may suggest that testosterone acts on striatal dopamine pathways to motivate risk-taking when anticipating reward of a potential status gain, specifically in those with low baseline dopamine availability. Future studies are required to replicate and test if this relation holds using larger samples.

It is important to note that, first, our sample only included men because the pharmacokinetics of the testosterone gel is only known in men. Although sex/gender differences for risk-taking are thought to be small (for review see Fisk et al., 2017), future research will be required to test whether our effects generalize to females. Second, to compare high and low-ranked individuals on risk preferences for identical risk choices and status outcomes (i.e. magnitudes, change in positions), high and low-ranked participants were placed in the middle of the new hierarchy containing only players of the same rank category. One possibility is that this induced an unstable hierarchy and a perceived small loss (gain) in status in the high (low) ranked participants. Consequently, testosterone may have reinforced risktaking in the high-ranked participants to "redeem" their top position, but inhibited risk-taking in the low-ranked to secure the middle position and avoid further status loss, consistent with previous findings (Losecaat Vermeer et al., 2020).

Concluding, exogenous testosterone increased risk-taking for status, but not for options without direct status implications, in men with high but not low social status. These findings provide important 
insight for research in the field and theoretical models of testosterone in risk-taking as a strategy for status-seeking.

\section{Acknowledgements}

We thank Isabelle Krol, Christina Fasching, Eric Förster, Katharina Hirning, Nace Mikus, Lisa Rosenberger, Sebastijan Veselic for assistance in data collection, Romana Raab (FDZ) for technical assistance in genotyping, and Igor Riecansky for medical support.

\section{Open practices statement}

The current design and analysis plans were not formally preregistered.

\section{References}

Apicella, C. L., Carré, J. M., \& Dreber, A. (2015). Testosterone and Economic Risk Taking: A Review. Adaptive Human Behavior and Physiology, Vol. 1, pp. 358-385. https://doi.org/10.1007/s40750-014-0020-2

Apicella, C. L., Dreber, A., \& Mollerstrom, J. (2014). Salivary testosterone change following monetary wins and losses predicts future financial risk-taking. Psychoneuroendocrinology, 39(1), 58-64. https://doi.org/10.1016/j.psyneuen.2013.09.025

Archer, J. (2006). Testosterone and human aggression: An evaluation of the challenge hypothesis. Neuroscience and Biobehavioral Reviews, 30(3), 319-345. https://doi.org/10.1016/j.neubiorev.2004.12.007

Bates, D., Mächler, M., Bolker, B., \& Walker, S. (2015). Fitting Linear Mixed-Effects Models Using Ime4. Journal of Statistical Software, 67(1), 1-48. https://doi.org/10.18637/jss.v067.i01

Boksem, M. A. S., Mehta, P. H., Van den Bergh, B., van Son, V., Trautmann, S. T., Roelofs, K., ... Sanfey, A. G. (2013). Testosterone inhibits trust but promotes reciprocity. Psychological Science, 24, 2306-2314. https://doi.org/10.1177/0956797613495063

Carré, J. M., Geniole, S. N., Ortiz, T. L., Bird, B. M., Videto, A., \& Bonin, P. L. (2017). Exogenous Testosterone Rapidly Increases Aggressive Behavior in Dominant and Impulsive Men. Biological Psychiatry, 82(4), 249-256. https://doi.org/10.1016/j.biopsych.2016.06.009

Casto, K. V., Edwards, D. A., Akinola, M., Davis, C., \& Mehta, P. H. (2020). Testosterone reactivity to competition and competitive endurance in men and women. Hormones and Behavior, 104665. https://doi.org/10.1016/j.yhbeh.2019.104665

Cueva, C., Roberts, R. E., Spencer, T., Rani, N., Tempest, M., Tobler, P. N., ... Rustichini, A. (2015). Cortisol and testosterone increase financial risk taking and may destabilize markets. Scientific Reports, 5(1), 11206. https://doi.org/10.1038/srep11206

de Souza Silva, M. A., Mattern, C., Topic, B., Buddenberg, T. E., \& Huston, J. P. (2009). Dopaminergic 
and serotonergic activity in neostriatum and nucleus accumbens enhanced by intranasal administration of testosterone. European Neuropsychopharmacology: The Journal of the European College of Neuropsychopharmacology, 19(1), 53-63. https://doi.org/10.1016/j.euroneuro.2008.08.003

Delgado, M. R., Schotter, A., Ozbay, E. Y., \& Phelps, E. a. (2008). Understanding overbidding: using the neural circuitry of reward to design economic auctions. Science (New York, N.Y.), 321(5897), 1849-1852. https://doi.org/10.1126/science.1158860

Derntl, B., Pintzinger, N., Kryspin-Exner, I., \& Schöpf, V. (2014). The impact of sex hormone concentrations on decision-making in females and males. Frontiers in Neuroscience, 8, 352. https://doi.org/10.3389/fnins.2014.00352

Eisenegger, C., Haushofer, J., \& Fehr, E. (2011). The role of testosterone in social interaction. Trends in Cognitive Sciences, 15(6), 263-271. https://doi.org/10.1016/j.tics.2011.04.008

Eisenegger, C., Kumsta, R., Naef, M., Gromoll, J., \& Heinrichs, M. (2017). Testosterone and androgen receptor gene polymorphism are associated with confidence and competitiveness in men. Hormones and Behavior, 92, 93-102. https://doi.org/10.1016/j.yhbeh.2016.09.011

Eisenegger, C., Naef, M., Snozzi, R., Heinrichs, M., \& Fehr, E. (2010). Prejudice and truth about the effect of testosterone on human bargaining behaviour. Nature, 463(7279), 356-359. https://doi.org/10.1038/nature08711

Eisenegger, C., von Eckardstein, A., Fehr, E., \& von Eckardstein, S. (2013). Pharmacokinetics of testosterone and estradiol gel preparations in healthy young men. Psychoneuroendocrinology, 38(2), 171-178. https://doi.org/10.1016/j.psyneuen.2012.05.018

Ermer, E., Cosmides, L., \& Tooby, J. (2008). Relative status regulates risky decision making about resources in men: evidence for the co-evolution of motivation and cognition. Evolution and Human Behavior, 29(2), 106-118. https://doi.org/10.1016/j.evolhumbehav.2007.11.002

Fisk, S. R., Miller, B. J., \& Overton, J. (2017). Why social status matters for understanding the interrelationships between testosterone, economic risk-taking, and gender. Sociology Compass, 11(3), e12452. https://doi.org/10.1111/soc4.12452

Geniole, S. N., \& Carré, J. M. (2018). Human social neuroendocrinology: Review of the rapid effects of testosterone. Hormones and Behavior, 104(February), 192-205.

https://doi.org/10.1016/j.yhbeh.2018.06.001

Geniole, S. N., Procyshyn, T. L., Marley, N., Ortiz, T. L., Bird, B. M., Marcellus, A. L., ... Carré, J. M. (2019). Using a Psychopharmacogenetic Approach To Identify the Pathways Through Whichand the People for Whom-Testosterone Promotes Aggression. Psychological Science, 1-14. https://doi.org/10.1177/0956797619826970

Goudriaan, A. E., Lapauw, B., Ruige, J., Feyen, E., Kaufman, J. M., Brand, M., \& Vingerhoets, G. (2010). The influence of high-normal testosterone levels on risk-taking in healthy males in a 1-week letrozole administration study. Psychoneuroendocrinology, Vol. 35, pp. 1416-1421. https://doi.org/10.1016/j.psyneuen.2010.04.005

Heinz, A. (2000). Genotype Influences In Vivo Dopamine Transporter Availability in Human Striatum. Neuropsychopharmacology, 22(2), 133-139. https://doi.org/10.1016/S0893-133X(99)00099-8

Hermans, E. J., Bos, P. A., Ossewaarde, L., Ramsey, N. F., Fernandez, G., \& van Honk, J. (2010). Effects of exogenous testosterone on the ventral striatal BOLD response during reward anticipation in healthy women. Neuroimage, 52(1), 277-283. https://doi.org/10.1016/j.neuroimage.2010.04.019

Losecaat Vermeer, A. B., Boksem, M. A. S., \& Sanfey, A. G. (2014). Neural mechanisms underlying context-dependent shifts in risk preferences. Neurolmage, 103, 355-363. 
https://doi.org/10.1016/j.neuroimage.2014.09.054

Losecaat Vermeer, A. B., Krol, I., Gausterer, C., Wagner, B., Eisenegger, C., \& Lamm, C. (2020). Exogenous testosterone increases status-seeking motivation in men with unstable low social status. Psychoneuroendocrinology, 113, 104552. https://doi.org/10.1016/j.psyneuen.2019.104552

Losecaat Vermeer, A. B., Riečanský, I., \& Eisenegger, C. (2016). Competition, testosterone, and adult neurobehavioral plasticity. In Progress in Brain Research (pp. 213-238). https://doi.org/10.1016/bs.pbr.2016.05.004

Mazur, A., \& Booth, A. (1998). Testosterone and dominance in men. The Behavioral and Brain Sciences, 21(3), 353-363; discussion 363-97. Retrieved from http://www.ncbi.nlm.nih.gov/pubmed/10097017

Mehta, P. H., \& Josephs, R. a. (2006). Testosterone change after losing predicts the decision to compete again. Hormones and Behavior, 50, 684-692. https://doi.org/10.1016/j.yhbeh.2006.07.001

Mehta, P. H., Son, V. Van, Welker, K. M., Prasad, S., Sanfey, A. G., Smidts, A., \& Roelofs, K. (2015). Exogenous testosterone in women enhances and inhibits competitive decision-making depending on victory-defeat experience and trait dominance. Psychoneuroendocrinology, 60, 224-236. https://doi.org/10.1016/j.psyneuen.2015.07.004

Mehta, P. H., Welker, K. M., Zilioli, S., \& Carré, J. M. (2015). Testosterone and cortisol jointly modulate risk-taking. Psychoneuroendocrinology, 56, 88-99. https://doi.org/10.1016/j.psyneuen.2015.02.023

Nadler, A., Jiao, P., Johnson, C. J., Alexander, V., \& Zak, P. J. (2017). The Bull of Wall Street: Experimental Analysis of Testosterone and Asset Trading. Management Science, mnsc.2017.2836. https://doi.org/10.1287/mnsc.2017.2836

Schneewind, K. A., \& Graf, J. (1998). 16-Persönlichkeits-Faktoren-Test. Revision (16 PF-R). In Deutsche Ausgabe des 16 PF (5th ed., pp. 1-133). Huber.

Stanton, S. J. (2017). The role of testosterone and estrogen in consumer behavior and social \& economic decision making: A review. Hormones and Behavior, 92, 155-163. https://doi.org/10.1016/j.yhbeh.2016.11.006

Stanton, S. J., Mullette-Gillman, O. A., McLaurin, R. E., Kuhn, C. M., LaBar, K. S., Platt, M. L., \& Huettel, S. A. (2011). Low- and high-testosterone individuals exhibit decreased aversion to economic risk. Psychological Science, 22(4), 447-453. https://doi.org/10.1177/0956797611401752

Tversky, A., \& Kahneman, D. (1981). The framing of decisions and the psychology of choice. Science (New York, N.Y.), 211(4481), 453-458. Retrieved from http://www.ncbi.nlm.nih.gov/pubmed/7455683

van Honk, J., Schutter, D. J. L. G., Hermans, E. J., Putman, P., Tuiten, A., \& Koppeschaar, H. (2004). Testosterone shifts the balance between sensitivity for punishment and reward in healthy young women. Psychoneuroendocrinology, 29(7), 937-943. https://doi.org/10.1016/j.psyneuen.2003.08.007

Welker, K. M., Goetz, S. M. M., \& Carré, J. M. (2015). Perceived and experimentally manipulated status moderates the relationship between facial structure and risk-taking. Evolution and Human Behavior, 36(6), 423-429. https://doi.org/10.1016/j.evolhumbehav.2015.03.006

Welker, K. M., Roy, A. R. K., Geniole, S., Kitayama, S., \& Carré, J. M. (2019). Taking risks for personal gain: An investigation of self-construal and testosterone responses to competition. Social Neuroscience, 14(1), 99-113. https://doi.org/10.1080/17470919.2017.1407822

Wu, Y., Liu, J., Qu, L., Eisenegger, C., Clark, L., \& Zhou, X. (2016). Single dose testosterone 


\section{7}

administration reduces loss chasing in healthy females. Psychoneuroendocrinology, $71,54-57$. https://doi.org/10.1016/j.psyneuen.2016.05.005

Zethraeus, N., Kocoska-Maras, L., Ellingsen, T., Von Schoultz, B., Hirschberg, A. L., \& Johannesson, M. (2009). A randomized trial of the effect of estrogen and testosterone on economic behavior. Proceedings of the National Academy of Sciences of the United States of America, 106(16), 6535-6538. https://doi.org/10.1073/pnas.0812757106

Zitzmann, M., \& Nieschlag, E. (2003). The CAG repeat polymorphism within the androgen receptor gene and maleness1. International Journal of Andrology, 26(2), 76-83. https://doi.org/10.1046/j.1365-2605.2003.00393.x 
528

529

530

531

532

533

534

535

536

537

538

539

540

541

542

543

544

545

546

547

548

549

550

551

552

553

554

\section{Supplementary online material}

Mood

Drug administration did not influence self-reported mood, alertness and calmness (multidimensional mood questionnaire, Steyer, Schwenkmezger, Notz, \& Eid, 1994) (all $F$ values $<1$ ). From pre- to postadministration alertness decreased $(F(1,164)=9.77, p=.002)$, calmness increased $(F(1,164)=10.97, p$ $=.001)$, but participant's mood did not statistically change $(F(1,164)=2.11, p=.148)$.

\section{Hormone analysis}

Saliva samples analyzed for testosterone and hydrocortisone using liquid chromatography tandem mass spectrometry (LCMS/MS), with an Agilent 6460 with electrospray ionization in positive mode coupled to a 1290 UHPLC system. Collision energy was optimized for specific MRM transitions of Hydrocortisone $(363.2 / 121.1 \mathrm{~m} / \mathrm{z} ; 363.2 / 91.1 \mathrm{~m} / \mathrm{z})$, Testosterone $(289.2 / 109.1 ; 289.2 / 97.1 \mathrm{~m} / \mathrm{z})$, 2,3,4-13C3-Hydrocortisone (366.2/124 m/z) and 2,3,4-13C3-Testosterone (292.2/100 m/z). Agilent Poroshell 120 EC-C18 was used for chromatographic separation under reversed phase conditions. For the internal standard preparation and internal standard mixture was prepared containing 2,3,4-13C3Hydrocortisone; 2,3,4-13C3-Testosterone, 2,4,16,16,17-d5-17b-Estradiol and 2,4,16,16-d4-Estron with a final concentration of $5 \mathrm{ng} / \mathrm{mL}$ each. Samples were prepared by adding $100 \mu$ internal standards ( 5 $\mathrm{ng} / \mathrm{mL}$ ) to $500 \mu \mathrm{l}$ plasma or salvia and the steroids were extracted using $4 \mathrm{~mL}$ MTBE. After 10 minutes overhead shacking, samples were centrifuged for 5 minutes at $3000 \mathrm{rpm}$ and the top MTBE layer was transferred to a test tube. MTBE was evaporated using a centrivap concentrator at $40^{\circ} \mathrm{C}$ (Labconco). The residual sample was then re-dissolved in methanol and analyzed by LC-MS/MS.

\section{Genotyping}

Buccal swabs were collected using sterile cotton swabs (Sarstedt AG, Germany) and stored at room temperature. DNA was extracted using the QIAamp DNA Mini kit (Qiagen, Hilden, Germany) following 
555

556

557

558

559

560

561

562

563

564

565

566

567

568

569

570

571

572

573

the kit's protocol: "DNA Purification from Buccal Swabs" and eluted in a final volume of $50 \mu \mathrm{L}$ of QIAamp buffer AE (Qiagen, Hilden, Germany). Human DNA was quantified using the Applied Biosystems (AB) 7500 real-time PCR system (Thermo Fisher Scientific, Waltham, MA) and the Quantifiler HP (Human Plus) Quantification Kit (AB) following manufacturer's recommendations. Both, androgen receptor (AR)CAGn and DAT1 VNTR were simultaneously co-amplified in a single tube reaction. Accordingly, template DNA (10 ng per sample) was subjected to 35 cycles of PCR amplification performed in a total volume of $25 \mu \mathrm{L}$ consisting of $1 \times$ GeneAmp PCR Buffer (AB), $0.25 \mathrm{mM}$ each dNTP, $0.5 \cup$ AmpliTaq Gold Polymerase (AB) and target specific primers (including fluorescently-labeled forward primers, details are provided in Table S1). The following thermal protocol was applied using the Veriti 96-well thermal cycler (AB): 35 cycles at $95^{\circ} \mathrm{C}$ for 30 seconds, $55^{\circ} \mathrm{C}$ for 1 minute, and $72{ }^{\circ} \mathrm{C}$ for 1 minute. Before the first cycle, an initial denaturation ( $95^{\circ} \mathrm{C}$ for 5 minutes) was included, and the last cycle was followed by a final extension step at $72{ }^{\circ} \mathrm{C}$ for 45 minutes. Aliquots of PCR products were diluted with Hi-Di formamide (AB), mixed with internal lane standard LIZ 600 v.2 (AB) and separated on the ABI 3500 Genetic Analyzer applying standard conditions. The number of repeats predicted by the GeneMapper ID-X software (AB) was in full agreement to the actual repeats determined by direct sequencing of PCR products using the BigDye Terminator Sequencing Kit v3.1 (AB) in selected DNA samples.

Table S1. Panel of loci and primer sets used for the typing of repeat length polymorphisms

\begin{tabular}{|c|c|c|c|c|c|}
\hline Marker & Location $^{\mathrm{a}}$ & Primer sequence $5^{\prime}-3^{\prime}{ }^{b}$ & Dye & Orientation & Conc. $(\mathrm{nM})^{\mathrm{c}}$ \\
\hline$A R(C A G) n$ & chrX:67545237-67545434(+) & $\begin{array}{l}\text { CGCGAAGTGATCCAGAACC } \\
\text { GTTTCTAGAACCATCCTCACCCTGCT }\end{array}$ & 6-FAM & $\begin{array}{l}\text { forward } \\
\text { reverse }\end{array}$ & 200 \\
\hline DAT1 VNTR & chr5:1393559-1394008(-) & $\begin{array}{l}\text { TGTGGTGTAGGGAACGGCCTGAGA } \\
\text { TGTTGGTCTGCAGGCTGCCTGCAT }\end{array}$ & 6-FAM & $\begin{array}{l}\text { forward } \\
\text { reverse }\end{array}$ & 400 \\
\hline
\end{tabular}

${ }^{a}$ Chromosome number and genomic location of targeted sequence (orientation provided in brackets)

575 according to UCSC version hg38 (http://genome.ucsc.edu/)

576 b The non-specific primer tail is underlined in Italics

$577{ }^{c}$ The final primer concentrations in the reaction mix 
The model included 158 participants, as some samples $(n=8)$ were not analyzable due to low quality or

587
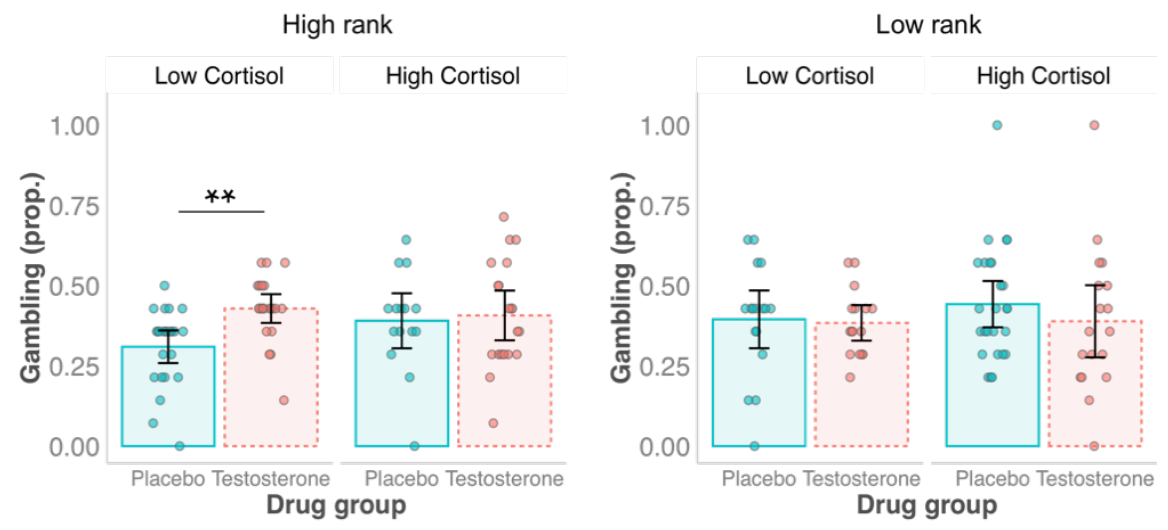

Figure S1. Risk-taking for status outcomes, split by rank and baseline cortisol (based on median split). Every dot represents participants' mean. Error bars represent $95 \% \mathrm{Cl}$. Asterisks indicate significant difference between $\operatorname{drug}(* * p<.01)$.

\section{Trait dominance}

As shown in Table S3, trait dominance did not influence risk-taking for money nor for status, nor did it interact with drug or rank. The main effect of rank when gambling for money remained significant $(p=.027)$. For status, the conditional effect of testosterone and rank reduced to marginal significance (drug x rank, $p=.056)$.

\section{CAG-repeat length}

As shown in Table S3, CAG-repeat length did not influence risk-taking for money nor for status, nor did it interact with drug and rank. The main effect of rank when gambling for money $(p=.018)$, and the drug x rank interaction for status $(p=.029)$ remained significant. 


\section{DAT1 genotype}

604

605

606

607

608

609

610

611

612

613

614

615

616

617

618

619

620

621

622

We included data from the 10/10 (testosterone $n=48$, placebo $n=46$ ) and 9/10-carriers (testosterone $n=29$, placebo $n=27$ ), as they represented the largest sample (total $n=150$ ), similar to other work (Eisenegger et al., 2013; Heinz, 2000). Sixteen participants not included consisted of 9/9-carriers (testosterone $n=3$, placebo $n=8$ ), 10/11-carriers (testosterone $n=2$, placebo $n=2$ ), and one 9/11-carrier (placebo).

As shown in Table S3, DAT1 genotype did not influence risk-taking for money nor for status, nor did it interact with drug and rank. The main effect of rank when gambling for money was marginal significant $(p=.051)$.

For status, DAT1 interacted with drug to predict risk-taking $(p=.035)$. Although not reaching statistical significance at post-hoc comparisons, decomposing the interaction suggest that $10 / 10$ carriers with testosterone (vs. placebo) were almost twice more likely to take risk ( $\mathrm{OR}=1.97, \mathrm{SE}=0.85$, $95 \% \mathrm{Cl}=[0.85 ; 4.59], \mathrm{z}=1.57, p=.116)$, whereas $9 / 10$-carriers with testosterone (vs. placebo) seem $56 \%$ less likely to take risk (OR=0.44, $\mathrm{SE}=0.25,95 \% \mathrm{Cl}=[0.15 ; 1.32], \mathrm{z}=-1.47, p=.143)$. Including DAT1 resulted in a marginal significant testosterone by rank effect $(p=.081)$.

Importantly, when including baseline cortisol, dominance, CAG-repeat and DAT1 as covariates in one model, the conditional effect of testosterone and rank on risk-taking for status was significant $(\mathrm{OR}=0.67,95 \% \mathrm{Cl}=[0.47,0.95], \mathrm{z}=-2.25, p=.024)$, whereas the main effect of rank for monetary gambles seemed less robust $(z=-1.51, p=.130)$. To note, this model could only be analyzed on a subset of the sample ( $n=142)$, which reduced statistical power. 
623 Table S3. Results of generalized mixed effects models for Money and for Status, for each moderator

\begin{tabular}{|c|c|c|c|c|c|c|c|c|c|c|}
\hline \multirow{2}{*}{$\begin{array}{l}\text { Baseline cortisol }(\mathbf{n}=\mathbf{1 5 8}) \\
\text { Predictors }\end{array}$} & \multicolumn{5}{|c|}{ Risk-taking for money } & \multicolumn{5}{|c|}{ Risk-taking for status } \\
\hline & OR & $S E$ & $95 \% \mathrm{Cl}$ & $z$ & $p$ & OR & $S E$ & $95 \% \mathrm{Cl}$ & $z$ & $p$ \\
\hline Intercept & 0.27 & 0.27 & $0.16,0.45$ & -4.86 & $<.001$ & 0.18 & 0.21 & $0.12,0.27$ & -8.09 & $<.001$ \\
\hline Rank & 0.65 & 0.23 & $0.41,1.02$ & -1.88 & .061 & 0.88 & 0.17 & $0.63,1.22$ & -0.78 & .433 \\
\hline Drug & 0.82 & 0.23 & $0.52,1.29$ & -0.87 & .387 & 1.14 & 0.17 & $0.82,1.58$ & 0.76 & .449 \\
\hline Cortisol log (t0) & 1.23 & 0.36 & $0.60,2.51$ & 0.56 & .573 & 1.09 & 0.27 & $0.64,1.84$ & 0.31 & .757 \\
\hline Expected Value & 7.62 & 0.15 & $5.72,10.15$ & 13.88 & $<.001$ & 1.16 & 0.01 & $1.14,1.19$ & 13.62 & $<.001$ \\
\hline Rank* Drug & 1.22 & 0.23 & $0.78,1.92$ & 0.87 & .386 & 1.47 & 0.17 & $1.05,2.04$ & 2.25 & .024 \\
\hline Rank * Cortisol log (t0) & 1.24 & 0.36 & $0.61,2.52$ & 0.59 & .555 & 1.25 & 0.26 & $0.74,2.10$ & 0.84 & .398 \\
\hline Drug * Cortisol log (t0) & 0.60 & 0.36 & $0.30,1.22$ & -1.41 & .159 & 0.94 & 0.27 & $0.55,1.59$ & -0.24 & .810 \\
\hline Rank $*$ Drug $*$ Cortisol log (t0) & 0.83 & 0.36 & $0.41,1.69$ & -0.50 & .614 & 0.73 & 0.26 & $0.43,1.23$ & -1.18 & .237 \\
\hline Marginal R2 / Conditional R2 & \multicolumn{5}{|c|}{$0.594 / 0.913$} & \multicolumn{4}{|c|}{$0.662 / 0.892$} & \\
\hline Trait dominance $(n=166)$ & \multicolumn{5}{|c|}{ Risk-taking for money } & \multicolumn{5}{|c|}{ Risk-taking for status } \\
\hline Predictors & $O R$ & $S E$ & $95 \% \mathrm{Cl}$ & $z$ & $p$ & $O R$ & $S E$ & $95 \% \mathrm{Cl}$ & $z$ & $p$ \\
\hline Intercept & 0.29 & 0.28 & $0.17,0.50$ & -4.45 & $<.001$ & 0.18 & 0.22 & $0.12,0.28$ & -7.82 & $<.001$ \\
\hline Rank & 0.58 & 0.24 & $0.36,0.94$ & -2.22 & .027 & 0.83 & 0.18 & $0.58,1.19$ & -1.02 & .309 \\
\hline Drug & 0.82 & 0.24 & $0.51,1.31$ & -0.84 & .401 & 1.22 & 0.18 & $0.86,1.74$ & 1.13 & .259 \\
\hline Trait dominance & 1.00 & 0.01 & $0.98,1.02$ & 0.13 & .899 & 1.00 & 0.01 & $0.99,1.01$ & 0.08 & .937 \\
\hline Expected Value & 7.33 & 0.14 & $5.60,9.60$ & 14.49 & $<.001$ & 1.16 & 0.01 & $1.14,1.19$ & 13.95 & $<.001$ \\
\hline Rank* Drug & 1.39 & 0.24 & $0.86,2.23$ & 1.35 & .177 & 1.41 & 0.18 & $0.99,2.01$ & 1.91 & .056 \\
\hline Rank * Trait dominance & 1.00 & 0.01 & $0.98,1.02$ & -0.05 & .959 & 0.99 & 0.01 & $0.98,1.01$ & -0.70 & .487 \\
\hline Drug * Trait dominance & 0.99 & 0.01 & $0.98,1.01$ & -0.63 & .526 & 1.00 & 0.01 & $0.99,1.02$ & 0.44 & .660 \\
\hline Rank * Drug * Trait dominance & 1.01 & 0.01 & $0.99,1.03$ & 0.78 & .434 & 0.99 & 0.01 & $0.98,1.01$ & -0.86 & .387 \\
\hline Marginal $\mathrm{R}^{2}$ / Conditional $\mathrm{R}^{2}$ & \multicolumn{5}{|c|}{$0.592 / 0.910$} & \multicolumn{3}{|c|}{$0.661 / 0.893$} & & \\
\hline CAG-repeat polymorphism ( $n=166)$ & \multicolumn{5}{|c|}{ Risk-taking for money } & \multicolumn{5}{|c|}{ Risk-taking for status } \\
\hline Predictors & OR & $S E$ & $95 \% \mathrm{Cl}$ & $z$ & $p$ & OR & $S E$ & $95 \% \mathrm{Cl}$ & $z$ & $p$ \\
\hline Intercept & 0.29 & 0.26 & $0.17,0.48$ & -4.75 & $<.001$ & 0.17 & 0.21 & $0.11,0.25$ & -8.54 & $<.001$ \\
\hline Rank & 0.59 & 0.22 & $0.38,0.91$ & -2.38 & .018 & 0.85 & 0.17 & $0.61,1.18$ & -0.98 & .326 \\
\hline Drug & 0.89 & 0.22 & $0.58,1.37$ & -0.53 & .595 & 1.14 & 0.16 & $0.83,1.58$ & 0.81 & .415 \\
\hline CAG-repeat & 1.02 & 0.08 & $0.88,1.19$ & 0.32 & .748 & 0.99 & 0.06 & $0.88,1.10$ & -0.22 & .827 \\
\hline Expected Value & 7.38 & 0.14 & $5.63,9.68$ & 14.44 & $<.001$ & 1.16 & 0.01 & $1.14,1.19$ & 13.96 & $<.001$ \\
\hline Rank* Drug & 1.27 & 0.22 & $0.83,1.96$ & 1.10 & .272 & 1.43 & 0.17 & $1.04,1.98$ & 2.18 & .029 \\
\hline Rank * CAG-repeat & 0.91 & 0.08 & $0.78,1.05$ & -1.29 & .199 & 0.91 & 0.06 & $0.82,1.02$ & -1.62 & .104 \\
\hline Drug * CAG-repeat & 1.11 & 0.08 & $0.96,1.29$ & 1.42 & .155 & 0.97 & 0.06 & $0.87,1.09$ & -0.50 & .614 \\
\hline Rank * Drug * CAG-repeat & 1.09 & 0.08 & $0.94,1.27$ & 1.17 & .242 & 1.05 & 0.06 & $0.94,1.17$ & 0.83 & .405 \\
\hline Marginal $\mathrm{R}^{2} /$ Conditional $\mathrm{R}^{2}$ & \multicolumn{5}{|c|}{0.594 / 0.911} & \multicolumn{3}{|c|}{$0.663 / 0.893$} & & \\
\hline DAT1 genotype $(n=150)$ & \multicolumn{5}{|c|}{ Risk-taking for money } & \multicolumn{5}{|c|}{ Risk-taking for status } \\
\hline Predictors & $O R$ & $S E$ & $9 \% 5 \mathrm{Cl}$ & $z$ & $p$ & $O R$ & $S E$ & $95 \% \mathrm{Cl}$ & $z$ & $p$ \\
\hline Intercept & 0.31 & 0.28 & $0.18,0.54$ & -4.16 & $<.001$ & 0.18 & 0.22 & $0.11,0.27$ & -7.85 & $<.001$ \\
\hline Rank & 0.62 & 0.24 & $0.38,1.00$ & -1.95 & .051 & 0.82 & 0.18 & $0.57,1.16$ & -1.12 & .262 \\
\hline Drug & 0.80 & 0.24 & $0.50,1.29$ & -0.91 & .365 & 0.96 & 0.18 & $0.68,1.36$ & -0.21 & .835 \\
\hline DAT1 & 0.70 & 0.24 & $0.43,1.13$ & -1.46 & .144 & 0.73 & 0.18 & $0.52,1.04$ & -1.76 & .078 \\
\hline Expected Value & 7.38 & 0.15 & $5.54,9.83$ & 13.68 & $<.001$ & 1.16 & 0.01 & $1.14,1.19$ & 13.48 & $<.001$ \\
\hline Rank* Drug & 1.15 & 0.24 & $0.71,1.85$ & 0.57 & .569 & 1.36 & 0.18 & $0.96,1.93$ & 1.75 & .081 \\
\hline Rank * DAT1 & 1.03 & 0.24 & $0.64,1.66$ & 0.13 & .897 & 1.09 & 0.18 & $0.77,1.54$ & 0.49 & .623 \\
\hline Drug * DAT1 & 1.39 & 0.24 & $0.86,2.23$ & 1.34 & .179 & 1.46 & 0.18 & $1.03,2.06$ & 2.11 & .035 \\
\hline Rank * Drug * DAT1 & 1.44 & 0.24 & $0.89,2.32$ & 1.50 & .134 & 1.30 & 0.18 & $0.92,1.84$ & 1.48 & .138 \\
\hline Marginal $\mathrm{R}^{2}$ / Conditional $\mathrm{R}^{2}$ & \multicolumn{5}{|c|}{$0.596 / 0.910$} & \multicolumn{3}{|c|}{$0.671 / 0.893$} & & \\
\hline
\end{tabular}

624 Note: zero-to-sum contrast were applied (i.e. effect coding). Testosterone and high rank were coded as 1.

625 Continuous variables Expected value, baseline cortisol, trait dominance and CAG-repeat length were centered at

6260. 
627

628

629

630

631

632

633

634

635

636

\section{References}

Eisenegger, C., Pedroni, A., Rieskamp, J., Zehnder, C., Ebstein, R., Fehr, E., \& Knoch, D. (2013). DAT1 polymorphism determines L-DOPA effects on learning about others' prosociality. PloS One, 8(7), 1-7. https://doi.org/10.1371/journal.pone.0067820

Heinz, A. (2000). Genotype Influences In Vivo Dopamine Transporter Availability in Human Striatum. Neuropsychopharmacology, 22(2), 133-139. https://doi.org/10.1016/S0893-133X(99)00099-8

Steyer, R., Schwenkmezger, P., Notz, P., \& Eid, M. (1994). Theoretical analysis of a multidimensional mood questionnaire (MDBF). Testtheoretische Analysen Des Mehrdimensionalen Befindlichkeitsfragebogen (MDBF). 